\title{
Fosfatasas alcalinas en el estudio de la osteopenia del prematuro
}

\author{
Andrés Román N.'; John Wilson Sch'; Juan Pablo Beca I.; José M. Cortés E.'; \\ Vivianne Polette B. ${ }^{2}$; Ana María Espíndola A. ${ }^{3}$
}

\section{Serum calcium, phosphate, alcaline phosphatase and bone density in preterm newborn infants at 28 throughout 44 weeks postconceptional age}

\begin{abstract}
Serum calcium, phosphaie and alcaline phosphatases with its bone fraction were measured in ten 28 to 30 week gesiationat age preterm newborn intonls. All these mousurements were repealed at 32 , 36 and 44 weeks of postconceplionol age. Bone mineral contenl was also delermined al 36 and 44 week's postconceplional age by dual photon absciptiomeiry. Results were matched with those liom a contiol group of len 30 io 37 week's gestolion newboin infarits. Serum calcium and phosphale levels were similar in both groups, while alcaline phosphalase aclivilies al 32,36 and $\Delta 4$ weeks were signilicanlly higher in premalure infants than in conirols ip $<0$. ODO h). Lower bone minerai conients were ospciated to higher alcaline phosphatase activily $[1=0.77, p<0.0001)$ which was olmost completely given by the bone fraction isoenzime. No abnotmalities were detected by $x$-ray bone studies among preterm infonis. The uselulness of serum alcaline phosphatase measurement os well as the limilotions of bone radiographs in ihe clinical approoch of osleopenio of premalury were thus hightighled.
\end{abstract}

(Key wards: intant, premalue, osteopenio, bone density, obsortiomelry, pholon, serum colcium, phosphate, olcoline phosphalase.]

La disminución de la mineralización ósea del recién nacido prematuro, también denominada osteoperia o enfermedad ósea metabolica del prematuro, es una enfermedad de incidencia creciente en la medida en que mejora la sobrevida de los recién nacidos de muy bajo peso de nacimiento ${ }^{1.6 .}$ Estos recién nacidos, cuyos depósitos de calcio están disminuidos, son alimentados con fórmulas lácteas o alimentación parenteral con aportes de calcio, fosfatos y vitamina $D$ insuficientes $^{7-9}$. Además, son muy frecuentemente tratados con furosemida, que aumenta las pérdidas urinarias de calcio y contribuye al desarrollo de la enfermedad ${ }^{10,11}$. La evolución de la ostcopenia en la mayoría de los casos es subclínica, y puede dar sintomas tardíos, con fracturas patológicas.

El estudio del contenido mineral del hueso ${ }^{11-2 l}$, cuya utilidad en el manejo clínico individual es

3. Servicio de Recién Nacidos de Clínica Alemana.

2. Tecnólogo Médico. Laboratorio de Clínica Alemsna.

3. Enfermera. Servicio de Recién Nacidos de Clínica Alemana. discutible, es una herramienta fundamental en el diseño de formulas enterales y parenterales que aporten suficientes nutrientes para prevenir el desarrollo de la enfermedad ${ }^{22-25}$. La radiología presta poca utilidad porque su interpretación es subjetiva y detecta signos tardios. La medición de las fosfatasas alcalinas en el suero representa un metodo más sensible y de uso clínico más generalizado como elemento de apoyo diagnóstic $^{26,27}$. La densitometría ósea de doble fotón ${ }^{16}$, técnica de desarrollo y aplicación recientes en nuestro país, ha aumentado la sensibilidad y la precisión diagnóstica en el estudio de este trastorno.

Con el propósito de valorar la importancia de la fosfabasa alcalina en el diagnóstico y evolución de la osteopenia del prematuro, se diseñó un estudio prospectivo para comparar la actividad de fosfatasas alcalinas totales (FA) y su fracción ósea (FAO) en prematuros de 28 a 30 semanas de edad gestacional y en un grupo control de niños nacidos a las 36 a 37 semanas de gestación, con respecto a los resultados obtenidos con radiología y densitometría osea. 


\section{Material y Método}

El grupo de estudio estuvo constituido por 10 rccién nacidos de pretérmino de 28 a 30 semanas de edad gestacioral, ingresados entre mayo de 1990 y marzo de 199] a los servicios de neonatología de la Clínica Alemana de Santiago y del Hospital Parroquial de San Bemardo. Se incluyó a todos los niños con estas características, independientemente de otras afecciones. Los niños fucron alimentados con leche matema o formula láctea para prematuros (\$26 prematuIos (R)) con 80 y $40 \mathrm{mg}$ de calcio y fosfato por $100 \mathrm{ml}$ de fómula, respectivamente. En lodos se midió calcemia, fos. femia, fosfatasas alcalinas totales (FA) y su fracción ósca (FAO) a las 32, 36 y 44 semanas de edad gestacional posiconcepcional. Se sealizó, adenás, radiografía esquelélica en ocho de los diez niños y densitometría ósea a las 36 y 44 semanas comegidas en todos los casos.

El grupo control estuvo formado por 10 recién nacidos de 36 a 37 semanas de cdad gestacional obstétrica, concordante con la evaluación pediatrica de la cdaó gestacional, libres de enfermedades, alimentados con leche materna exclusiva. En este grupo se deteminó calcemia, fos[emia, FA, FAD, y densitomelría ósea durante la primera semana de vida.

Para la deteminación de calcemia se utilizó el método de $O$-cresolftaleína-complex ona, sin desproteinización, y para la fosfemia la lécnica de LV con molibdato de amonio en medio ácido. Para el estudio de las fosfalasas alcalinas se utilizó el método enzimático con tanpón AMP y la fracción ósea por precipitación con lectina de gemen de trigo ${ }^{2 k}$ (Bochringer Mannheim).

La densitometría ósea se efectuó con densitómetro Norland de doble fotón, mudelo 2600. Se hjcieron determi. naciones de cuerpo entero y se calculo contenido mineral tolal (TBM), TBMfpeso y densidad ósea total (BMD) estudio radiológico consistió en radiografía de huesos latgos en la que sc buscó signos de desmineralızación.

Para cada uno de los aspectos estudiados, con excepción de la radiología, se calcularon promedios y desyiaciones estándar, ulilizando la prueba de I de Siudent como para la significación de los resultados.

Los padres fueton intormados y se les solicito su consentimiento por escrito previo al ingreso de los pacientes en el estudio.

\section{Resultados}

El peso de nacimiento promedio fue $2833 \mathrm{~g}$, variando entre 2500 y $3290 \mathrm{~g}$ y la edad geslacional al nacer era 36,8 semanas en los controles. En el grupo de estudio, el promedio de peso de nacimiento era $1277 \mathrm{~g}$, variando entre 873 y 1585 , y la cdad gestacional promedio fue 29,1 semanas.

En el grupo control la calcemia fue $9,28 \pm$ $0,94 \mathrm{mg} / \mathrm{dl}$, fosfemia de $5,86 \pm 0,67 \mathrm{mg} / \mathrm{dl}$ y las fosfatasas alcalinas $216,2 \pm 71,3 \mathrm{U} / 1$ con fracción ósea 216,2 271,3 U/1. En los recién nacidos pretérmino, las calcemias fueron $9,88 \pm 0,73$ $\mathrm{mg} / \mathrm{dl}, 9,85 \pm 0,59 \mathrm{mg} / \mathrm{dl}$ y $10,44 \pm 0,57 \mathrm{mg} / \mathrm{dl}$, a las 32,36 y 44 semanas de edad postconcepcional y las fosfemias $6,10 \pm 0,73 \mathrm{mg} / \mathrm{dl}, 6,0$ $\pm 0,63 \mathrm{mg} / \mathrm{dl}$ y $6,23 \pm 1,03 \mathrm{mg} / \mathrm{dl}$ a las mismas semanas, sin diferencias estadísticamente significativas con el grupo control. En cambio, los valores de fosfatasa alcalina a las 32,36 y 44 semanas fueron $439 \pm 125,5 ; 471 \pm 126,5$ y 485 $\pm 143,7 \mathrm{U} / \mathrm{l}$ respectivamente y las correspondientes a la isoenzima ósea a las mismas semanas de edad gestacional corregida fueron de $400 \pm 102,6 ; 466 \pm 118,3$ y $469 \pm 144,9 \mathrm{U} / \mathrm{C}$ respectivamente, constituyendo la fracción osea casi la totalidad de la actividad de la fosfatasa alcalina global, todos ellos significativamente diferentes de los registrados en los niffos control $(p<0,0001)$.

El estudio radiológico, de ocho de los diez recién nacidos del grupo de prematuros, no mostró a las 36 y 44 semanas de edad gestacional corregida, signos de desmineralización en ninguno.

\section{Tabla}

Fosfatasas alcalinas séricas y densirometría ósea de diez recién nacidos de pretérmino ( 28 a 30 semanas $E G$ )

\begin{tabular}{|c|c|c|c|c|c|c|c|c|}
\hline & \multicolumn{2}{|c|}{ RN 36-37 sem } & \multirow{2}{*}{\multicolumn{2}{|c|}{$32 \mathrm{sem}$}} & \multirow{2}{*}{\multicolumn{2}{|c|}{$\begin{array}{l}\text { RN pretérmlno } 28 \text { - } 30 \mathrm{sem} \\
\mathbf{3 6} \mathrm{sem}\end{array}$}} & \multirow{2}{*}{\multicolumn{2}{|c|}{$44 \mathrm{sem}$}} \\
\hline & $\bar{x}$ & $\mathrm{DE}$ & & & & & & DE \\
\hline $\begin{array}{l}\text { Fosfatasas } \\
\text { alcalinas (U/) }\end{array}$ & 216,2 & 71,3 & 439 & 125,5 & 471 & 126,5 & 485 & 143,7 \\
\hline TBM/p $\quad(\mathrm{g})$ & 19,7 & 1,9 & 一 & - & 11,2 & 1,5 & 12,2 & 2,2 \\
\hline $\begin{array}{l}\text { Densidad } \\
\text { ósea }(\mathrm{g} / \mathrm{cm} \pm)\end{array}$ & 0.531 & 0,06 & - & - & 0,275 & 0,05 & 0,536 & 0,04 \\
\hline
\end{tabular}

TBM/p : masa ósea total/pcso 
La densidad osea, en cambio, mostró correlación negativa con las fosfacasas alcalinas, según se aprecia en la tabla y en la figura 1. El análisis de regresión lineal arrojo un indice de 0,77 como se observa en la figura $2(\mathrm{p}<0,0001)$.

\section{Comentario}

Se ha descrito retraso de la osificación en los recién nacidos prematuros alimentados con leche materna o con fớrmulas lácteas habituales, con respecto a la velocidad de osificación observada en la vida intrauterina. La evolución asintomática de la enfermedad hasta las etapas tardias y la falta de un método clínico práctico para ello, difjculta el diagnóstico de la osteopenia. Se ha empleado con frecuencia las fosfatasas alcalinas en el estudio de la osteopenia, pero su interpretación presenta también algunas dificultades, en particular en establecer los valores normales para recién nacidos prematuros con velocidad de crecimiento tan acelerada como la del último trimestre del embarazo. Sin embargo, se ha sugerido que concentraciones séricas de 500 $\mathrm{U} / \mathrm{l}$ indicarían osteopenia de cierta significación $^{30,11}$. Por otra parte, la diversidad de isoenzimas de fosfatasas alcalinas podría hacer dudar de su especificidad en el diagnóstico de enfermedad ósea.

Nuestro estudio muestra que, en nacidos pretérmino, las concentraciones séricas de fosfatasas alcalinas y su isoenzima ósea están elevadas desde las 32 semanas postconcepcionales, son signilicativamente mayores que en nifios de término y se mantienen sin variación hasta las 44 semanas de edad postconcepcional, estableciéndose una correlación negativa con la densidad osea. Sin embargo, pese a la severidad de las alteraciones de la mineralización mostradas por estos dos métodos en todos los prematuros, ninguno tuvo evidencias clínicas o radiológicas de enfermedad. La normalidad de las determinaciones de calcemia y fosfemia en ellos tampoco aportó elementos de apoyo al diagnóstico. Esto respalda el concepto de que ni la clínica ni la radiología son confiables en el diagnóstico de osteopenia del prematuro.

El hecho que casi el total de las fosfatasas alcalinas detectadas corresponde a la fracción ósea $(\bar{x} 96,5 \%)$ y, por otro lado, la existencia de correlación significativa entre su elevación y la

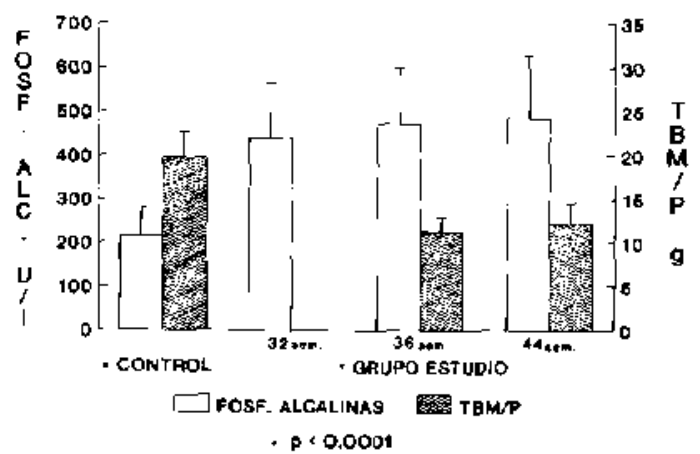

Fig. 1: Fosfatasas alcalinas y densitometría os ea en recien nacidos del grupo control (36 - $37 \mathrm{sem}$ ) y en el grupo de estudio ( $28-30$ sem) a las 32,36 y 44 sem de edad postconcepcional.

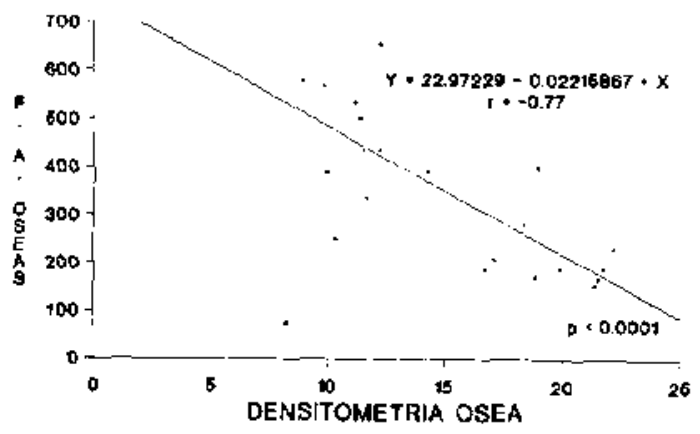

Fig. 2: Correlación entre la fracción óses de las fosfarasas alcalinas y la densitometría ósea en recién nacidos prematuros de $28 \cdot 30 \mathrm{sem}$.

disminución de la densidad ósea determinada por densitometría de doble fotón, reafirman la utilidad de la medición de las fosfatasas alcalinas plasmáticas en el diagnóstico de la osteopenia del prematuro. Los exámenes radiológicos de los huesos - como se habia descrito anteriormente y se comobora en nuestro estudio- no muestran alteraciones pese a la severidad de las anormalidades demostradas por las otras dos técnicas, lo que hace poco recomendable su uso en clínica.

Podemos concluir que las fosfatasas alcalinas totales, por corresponder casi completamente a la fracción ósea en el período neonatal, pueden ser usadas como elcmento para el diagnóstico de osteopenia. La coincidencia entre los resultados las concentraciones de fosfatasas alcalinas y los de densitometría ósea, permiten recomendar la primera de las técnicas como método de apoyo diagnostico de osteopenia. 


\section{Resumen}

Se midieron calcemia, fosfemia, fosfatasas alcalinas y la fracción ósea de estas en diez recién naciơos de pretérmino entre 28 y 30 semanas de edad gestacional al nacer y a las 32,36 y 44 semanas de edad gestacional corregida. Además se les determinó densidad ósea por densitometria de doble fotón y radiografía esquelética a las 36 y 44 semanas de edad gestacional corregida. Los resultados obtenidos fueron comparados con un grupo control de otros diez recién nacidos de 36 a 37 semanas de edad gestacional. Calcemias y fosfemias no mostraron diferencias significativas entre ambos grupos. Sin embargo, las concentraciones séricas de fosfatasas alcalinas a las 32,36 y 44 semanas fueron significativamente distintas que en el grupo control ( $p<0,0001)$. La disminución de la densidad ósea en los niños prematuros ( $\mathrm{p}<0,0001)$ se correlacionó con la elevación de las fosfatasas alcalinas $(r=0,77$; $p<0,0001$ ). Casi el total de las fosfatasas alcalinas medidas correspondía a la fracción ósea. La radiografía ósea no demostró alteraciones en los niños prematuros y sus controles, a pesar de los cambios descritos. Se demuestra la utilidad de la medición de FA como elemento diagnóstico en osteopenia y se corrobora la nula utilidad de la radiología ósea en la detección precoz de esta patología.

(Palabras clave: recién nacido, prematuro, osteopenia, densidad osea, densitometría de absorción, fotón, calcio sérico, fosfaro, fosfatasa alcalina.)

\section{Agradecimientos}

Agradecemos la valiosa colaboración de la Sra. Maria Gloria Icaza, del Departamento de Esladistica del LVTA. Universidad de Chile, por el análisis estadístico de los dalos; al Laboratorio Boehringer Mannhein que facilito los reactivos para el análisis bioquímico; al Laboratorio Wyeth que aportó la fómula láctea para prematuros.

\section{Referencias}

1. Forbes $G$ : Some remarks on bone mineralization. I Pediatr 1988; 113: 167-171.

2. Minton S, Steichen J, Tsang R: Bone mineral content intem and preterm appropiate-for-gestational-agc infants. I Pedialt 1979; 95: 1037-1042.

3. Steichen $J$, Gratton $T$. Tsang $R$ : Osteopenia of prematurity: the cause and possible treatment. J Pediatr 1980; $96: 528-534$.
4. Koo W, Gupto J, Nayanar V, Wilkinson M. Posen S: Skeletal changes in pretenm infants. Arch Dis Child 1982; $57: 447-452$.

5. James J, Congdon P, Truscolt J, Horsman A, Arthur R: Osteopenia of prematurity. Arch Dis Child 1986; 61: $871-876$

6. Horsman A, Ryan $S$, Congdon $P$, Truscott $J$, James $J$ : Osteopenia in extremely low birthweight infents. Arch Dis Child 1989; 64: $485-488$

7. Roberts $C$, Chan $G$, Folland $D$, Rayburn $C$, Jackson $R$ : Adequate bone mineralization in brest-fed infants. I Pediat 1981:99:192-196

8. Gross $S$ : Bone mineralization in preterm infants fed human milk with and without mineral supplementation. J Pediatr 1987; $111: 450-458$.

9. Mac Mahon P, Blair ME. Treweeke P. Kovar $f$ : Asscciation of mineral composition of neonatal intravenous feeding solutions and metabolic bone disease of prematurity. Arch Dis Child 1989; 64: 489-493.

10. Ryan S, Congdon P. Horsman A, James J. Truscott $A$, Arthur $R$ : Bone mineral content in broncopulmonary dysplasia. Arch Dis Child 1987; 62: 889-894.

11. Atkinson S, Shah $J, M c$ Gee C, Stecle D: Mineral excretion in premature infants receivig various diuretic therapies. J Pediatr 1988; $113 \div 540-545$.

12. Barden $H$, and Mazess $R$ : Bone densitometry in infauts. J Pediatr 1988: 113: 172-177.

13. Steichen J, Steichen $P$, and Tsang $R$ : Bone mineral content measurement in small infants by single -photon absorptiometry: Current metodologic issues. I Pediatr 1988: 113: 181-187.

14. Vykmeister $N$, and Linkhart $T$ : Measurcment of humerus and radius bone mineral content in the preterm and term infamt. J Pediatr 1988; 113: 188-195.

15. Petersen S, Gotfredsen A, and Kredsen F. Lean body mass in small for gestational age and appropjate for gestational age infants. J Pediatr 198B; 113: 886-889.

16. Petersen $S$, Golfredsen A, and Knudsen F: Total body bone mineral in light for gestalional age infants and appropiate for gestational age infants. Acta Paediatr Scabd 1989: $78: 347$.

17. Mazess $R$ : Bone densitomelry in infants. J Pediatr 1988: $113: 196-204$

18. Horsman A, Ryan SW, Congdon PJ, Truscoll JG, Simpson $M$ : Bone mineral content and body size preterm and full term infants. Arch Dis Child 1989; 64 1579-1586.

19. Minoweti $F$, and $T$ sang $R$ : Bone mineral content: Data analysis. J Pediat 1988; 113: 178-180.

20. Minton S, Steichen $f$, and Tsang $R$ : Decreased bone mineral content in Small-for-Gestational-Age infants compared with. Appropiate-for-Gestational-Age infams: Normal senum 25-Hidroxivitamin $D$ and decreasing parathyroid hormone. Pediatrics 1983; 71: 383-388.

21. Vyhmeister $N$, Linkhart T, Hay $S$, Baylink D, Ghosh $B$. Measurement of bone mineral content in the tem and pretem infant. AJDC 1987; 141: 506-510.

22. Greer $F$, Steichen $f$. Tsong $R$ : Effects of increased calcium, phosphorus and vitamin $D$ inlake on bone mineralization in very low-birth-weight infants fed formulas with policose and medium-chain triglicerides. J Pediatr 1982; 100: 951-955. 
23. Chan $G$, Leeper $L, B$ ook $L$ : Effects of soy formulas in mineral metabolism in term infants. AJDC 1987; 141: 527-530.

24. Sieichen $J$, Tsang $R$ : Bone mineralization and growth in term infants fed soy-based or cow milk-based formula. J Pediatr 1987; 110: 687-692.

25. Greer F, Mc Cormick A. Improved bone mineralization and growth in premature infants fed fortified own mother's milk. J Pediatr 1988; 112: $961-969$.

26. Moss D: Alkaline phosphatase isoenzymes. Clin Chem 1982; 28: 2007-2016.

27. Rosaiki $S$, Ying Foo A: Two new methods for separating and quantifying bone and liver alcaline phospha- tases isoenzymes in plasma. Clin Chem 1984: 30 : $1182-1186$.

28. Behr W, Barnert $J$ : Quantification of bone alcaline phosphatase in serum by precipitation with wheat-gemn lectin: a simplified method and its clinical plausibility. Clin Chem 1986; 32: 1960-1966.

29. Wilson S, Romon A, Beca JP, et al.: Osteopenia de niños nacidos antes de témino. Evaluación mediante densitometría ósen. Rev Chil Pediatr 1993; 64: 89-93.

30. Glass EV; Plasma alcaline phosphatasa activity in rickets of premaiury. Arch Dis Child 1982; 57: 373.

31. Kovar HYA- Plasma alcaline phosphatasa activity in the pretern neonate. Acta Paediatr Scand 198I; 70: 501 . 